\title{
Cytomegalovirus Reactivation in Ulcerative Colitis Patients
}

\author{
Minh Nguyen, ${ }^{1}$ Kara Bradford, ${ }^{1}$ Xiaolan Zhang, ${ }^{2}$ and David Q. Shih ${ }^{1,3}$ \\ ${ }^{1}$ Department of Medicine, Cedars Sinai Medical Center, Los Angeles, CA 90048, USA \\ ${ }^{2}$ Department of Gastroenterology, The Second Hospital of Hebei Medical University, Shijianzuang, Hebei 05000, China \\ ${ }^{3}$ Inflammatory Bowel and Immunobiology Research Institute, Cedars-Sinai Medical Center, Davis Building, Room 4066, \\ Los Angeles, CA 90048, USA
}

Correspondence should be addressed to David Q.Shih, david.shih@cshs.org

Received 14 August 2010; Accepted 15 November 2010

Academic Editor: T. Arakawa

Copyright (c) 2011 Minh Nguyen et al. This is an open access article distributed under the Creative Commons Attribution License, which permits unrestricted use, distribution, and reproduction in any medium, provided the original work is properly cited.

Ulcerative colitis (UC) patients are believed to have an increased risk for the colonic reactivation of cytomegalovirus (CMV) infection due to both inherent and iatrogenic factors. Numerous studies and case reports have described CMV infection as complicating the disease course of ulcerative colitis patients; the existing evidence suggests an association between the presence of CMV infection and increased colectomy and mortality rates in UC patients. Whether CMV is nonpathogenic with a tropism towards areas of dysplasia and inflammation in the colon of UC or plays an active role in pathogenesis is still debated. In this paper, we examine the existing evidence for the diagnosis and management of CMV infection in UC patients.

\section{Background}

Cytomegalovirus is a member of the Herpesviradae family, which also includes Epstein- Barr virus, herpes simplex virus, varicella-zoster virus, and herpesvirus 6,7 , and 8 [1]. The virus is transmitted through close personal contact with body fluids, including saliva, urine, blood, breast milk, semen, and transplanted organ tissue [1]. CMV infection is ubiquitous in developed nations, with laboratory evidence of prior infection in $40 \%-70 \%$ of the general adult population $[2,3]$. Immunocompromised patients may present with severe end-organ involvement as a manifestation of primary acute CMV infection $[1,4]$. In contrast, immunocompetent individuals with primary CMV infection are generally asymptomatic, only rarely develop colitis, encephalitis, myocarditis, and other organ-specific entities. In immunocompetent population, primary CMV infection is generally self-limited, and resolves to a state of lifelong latency. In the latent phase, the viral genome exists in an episomal circular form and does not replicate [5]. Latent CMV evades the host immune system, remaining dormant in myeloid progenitor cells and endothelial cells, without active viral replication or manifestation of clinical symptoms [6]. Latent infection carries no known increased morbidity or mortality risk. However, in the setting of immune activation, such as with inflammation, cytokines TNF-alpha and IFN-gamma can induce CMV-harboring monocytes to differentiate into macrophages [6-8]. This in turn may activate $\mathrm{CMV}$ replication and migration to inflammed tissue to further propagate infection $[6,7]$. CMV reactivation, seen clinically in acquired defects of cellular immunity, such as with immunosuppressant therapy, chemotherapy, bone marrow or solid organ transplantation, and HIV/AIDS, can lead to high disease activity and mortality [2].

\section{Increased Risk of CMV Reactivation in UC Patients}

Numerous case reports and prior studies have noted increased colonic CMV reactivation in ulcerative colitis patients [2, 4, 9-17]. A study of 21 idiopathic ulcerative colitis patients demonstrated a statistically significant higher proportion of patients with enteric tissue CMV, 57.1\% in UC compared to $14.3 \%$ in colorectal cancer patients [18]. The authors used the colorectal cancer group as a surrogate for the general population to conclude that UC carries an increased risk of CMV reactivation. A cross-sectional prospective study estimated that $\mathrm{CMV}$ in intestinal tissue biopsies were about 20 times more likely in UC patients 
than control individual with noninflammatory disease [3]. Similar studies have demonstrated increased CMV colonic reactivation in UC patients $[2,4,9-17]$. Limitations of these studies include the inclusion of only hospitalized or symptomatic UC patients and the lack of a true control population of healthy patients.

\section{Factors Contributing to CMV Reactivation in UC Patients}

This increased risk for reactivation of latent-CMV disease UC patients compared to the general population is thought to be due to both iatrogenic and inherent factors. For instance, multiple studies, including that of non UC posttransplant patients, have correlated the use of steroids and/or immunosuppressants to increased CMV reactivation rates [18-20]. After examining a variety of clinical and demographic factors, only systemic steroid use was found to be correlated to CMV infection [21]. Duration of steroid exposure was also an important factor contributing to CMV reactivation since use of steroids for greater than three months was associated with increased CMV rates among UC patients [22]. At this time however, the cumulative steroids dose that would predisposing to CMV reactivation has not been established.

Another risk factor for CMV reactivation is steroidrefractory disease. A case control study showed positive CMV immunohistochemistry (IHC) in the surgical specimen of 10 of $40(25 \%)$ patients with steroid-refractory UC and 1 of $40(2.5 \%)$ patients with nonrefractory UC $(P=.007)$ [10]. The authors estimated at least a 10 -fold increased incidence of colonic CMV among steroid-refractory patients and recommended detection of CMV should be part of the routine evaluation of steroid-refractory UC patients [10]. Similarly, another study found either CMV inclusion bodies and/or positive IHC in colonic biopsies in 6 out of 19 steroidrefractory UC patients but not in 75 inactive UC or steroidresponding UC patients [9]. Despite these accumulating evidences, steroid-refractory UC may simply be a surrogate marker of severe inflammation and not a direct contributor to $\mathrm{CMV}$ reactivation.

Association of cyclosporine, biologic therapy, and immunomodulator therapies to CMV reactivation in UC has also been studied. One study found that 18 of 23 UC patients developed active CMV infection after approximately 3 days of cyclosporine treatment [20]. A prospective study found active CMV disease in 5 out of 6 UC patients after 7-10 days of cyclosporine treatment [9].

To determine if there is any potential association between CMV infection and anti TNF-alpha use, 11 consecutive patients with inflammatory bowel disease were checked before and after a standard 3-infusion course of infliximab [23]. The authors did not find active CMV infection using conventional histology and IHC stains, suggesting that there is no increased risk of clinically significant CMV reactivation in the setting of TNF-alpha inhibitors [23]. More studies are needed to assess the relationship of anti TNF-alpha therapy to CMV reactivation including whether duration of anti TNF-alpha therapy is a risk factor to CMV reactivation.
At this time, there are conflicting results on the association of CMV reactivation with thiopurine drugs. Some studies found association of CMV reactivation with azathiopurine $[3,22,24,25]$. Other studies, however, did not show an association between CMV reactivation and azathiopurine [3,22]. Additional larger, controlled studies are needed to determine whether immunomodulators used in UC are linked to increased CMV reactivation.

Overall, these studies suggest that iatrogenic immunosuppression may contribute to CMV reactivation in UC patients. However, the presence of CMV reactivation in patients with little or no steroids/immunosuppressant exposure suggests that there are additional factors that contribute to the reactivation of CMV infection. One report reviewing the existing literature found that 7 of 27 IBD patients with CMV reactivation had less than one month of steroid use, and 5 patients had no prior exposure at all [2]. Similarly, another study found at least 5 case reports of UC patients developing CMV in steroid-naïve patients [12]. Investigations to identify other risk factors for CMV reactivation have failed to consistently correlate age, sex, time to diagnosis, number of prior flares, inflammatory markers, or nutritional status to increased CMV reactivation among UC patients $[3,9,21,25]$.

One factor that appears to correlate with CMV reactivation in UC patients independent of immunosuppression is the severity of disease itself. In a study involving 63 IBD patients of whom 61 were UC patients, pancolitis and histologic activity were significantly and independently correlated with CMV infection [25]. A Japanese study of 126 post-colectomy surgical specimens stratified by surgical indications found $25 \%$ of 32 patients labeled as "Severe" UC demonstrated histologic evidence of CMV, compared to $8.3 \%$ of 72 patients with "Refractory" UC and $0 \%$ of 22 patients for "Dysplasia or cancer" UC [26]. In this study, high steroid use is not found to be a risk factor for CMV infection [26].

\section{Diagnosis of CMV Reactivation in UC Patients}

Despite the recognition of increased risk of CMV reactivation in UC patients, the efficient and accurate diagnosis of active CMV infection in this population remains a challenge. CMV colitis shares many similar clinical features to an active UC flare. Patients may present with combination of fevers, malaise, anorexia, weight loss, abdominal pain, diarrhea, hematochezia, and tenesmus. Similarly, there are no endoscopic findings that have been reliably able to distinguish CMV colitis from active ulcerative colitis. The colonic appearance may include areas of erythema, ulceration, exudates, erosions, edema, and/or pseudotumors [36]. Studies examining elements of the presenting symptomatic complaints, initial laboratory data, and ensuing hospital course as predictors of CMV colitis versus active UC have not been systematically performed. Subanalysis of prior studies suggest that evidence of hematologic derangements secondary to bone marrow depression or severe hypokalemia on laboratory testing may indicate CMV involvement $[22,37]$. 


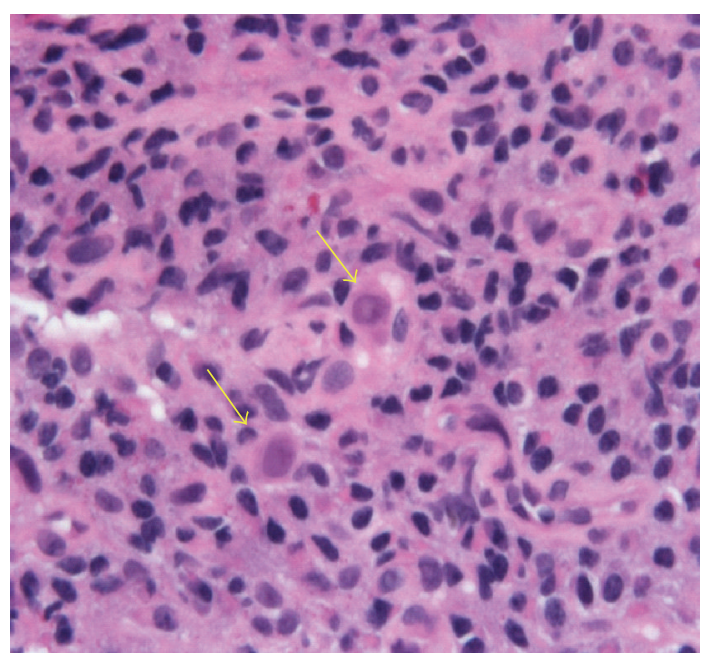

(a)

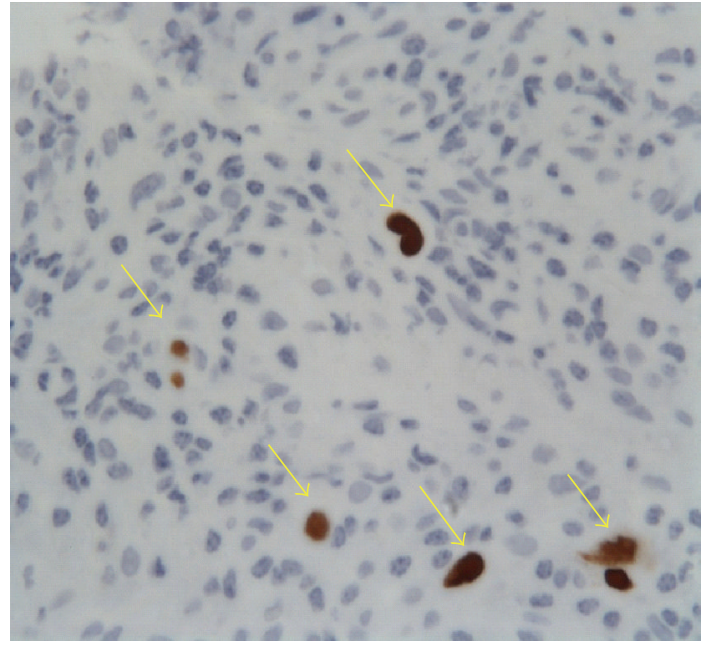

(b)

FIGURE 1: Histologic examination of CMV infection. (a) Haematoxylin and Eosin (H\&E) staining for CMV. CMV-infected cells are indicated by yellow arrows. Cells infected with CMV are larger, with a thickened nuclear membrane and granular intracytoplasmic inclusions. Typical "owl's eyes" or basophilic intranuclear inclusion bodies are representative of active CMV replicating nucleoprotein cores. (b) Immunohistochemistry (IHC) staining of CMV colitis. The use of IHC staining in histologic examination of CMV colitis can increase the diagnostic sensitivity. Typical IHC staining for enteric CMV is denoted by yellow arrows.

Others reports have suggested that clinical signs of fever, lymphadenopathy, splenomegaly, toxic megacolon and rapid clinical deterioration can help distinguish a superimposed CMV infection from a UC flare [19, 37]. However, these findings have not been independently validated.

CMV-specific laboratory testing has also been unreliable in diagnosing active CMV colitis. CMV serology, antigen testing, and DNA testing are useful for identifying prior exposure history, but can be difficult to interpret in the setting of active infection, and correlate poorly with active disease [36, 38]. Higher levels of quantitative DNA PCR have been suggested as indicative of active infection, but further studies are needed to elucidate whether CMV PCR levels correlate with active disease, response to therapy, or clinical outcomes. Experts believe that CMV appears to be a local process triggered by local proinflammatory cytokines as well as systemic immunosuppressant conditions, which could explain the poor predictive and correlative nature of serum CMV studies to active colitis [9]. At this time, the gold standard to diagnosing active CMV infection in UC patients requires histologic examination of involved tissue.

Histologic examination of diseased colon can show a variety of findings that indicate CMV reactivation. On Haematoxylin and Eosin (H\&E) staining, CMV-infected cells are typically 2 to 4 -fold larger than their surrounding cells and demonstrate a thickened nuclear membrane and granular intracytoplasmic inclusions (Figure 1(a)). Typical "owl's eyes," or basophilic intranuclear inclusion bodies surrounded by a clear halo, can be seen and are representative of active CMV replicating nucleoprotein cores (Figure 1(a)). In addition, special IHC staining of murine monoclonal antibodies specific to one of immediate early (IE) antigens of HMCV have been shown to increase the diagnostic sensitivity histologic examination for enteric CMV [36]. Studies have shown that up to $37.5 \%$ of patients with enteric CMV do not demonstrate any typical inclusions on H\&E staining, and that the addition of IHC staining can increase the sensitivity to $93 \%$ [36]. Typical IHC staining for enteric CMV is shown is Figure 1(b).

To date, the identification of either typical CMV inclusion bodies on routine staining or special immunohistochemical stains against CMV remains the most reliable method to diagnose active CMV colitis. The various diagnostic techniques to detect CMV are summarized in Table 1.

\section{Increased Morbidity and Mortality in UC Patients with CMV and Response to Antiviral Therapy}

The importance of prompt and proper recognition of CMV reactivation in UC patients has been highlighted by studies showing increased morbidity and mortality. A prospective observational study of 114 UC patients found that among patients with steroid-refractory disease, 3 of $6(50 \%)$ patients with evidence of CMV reactivation proceeded to colectomy, compared to 2 of $13(15 \%)$ steroid-refractory patients without CMV [9]. Another study of 63 patients, of which 61 were UC patients, found that 4 of $10(40 \%)$ patients with $\mathrm{CMV}$ required surgical intervention compared to 4 of 53 (7\%) UC patients without CMV; 3 of $10(30 \%)$ UC patients with CMV had fatal outcome, compared to 0 of $53(0 \%)$ UC patients without CMV [25].

Early detection of CMV reactivation in UC patients allows for therapeutic intervention with IV antiviral therapy, including ganciclovir or forscarnet, which has been 
TABLE 1: Summary of diagnostic techniques and its characteristics for CMV infection.

\begin{tabular}{|c|c|c|c|c|}
\hline Diagnostic tests & Pros & Cons & Sensitivity & Specificity \\
\hline Serum serology & $\begin{array}{l}\text { Can detect evidence of } \\
\text { prior infection }\end{array}$ & $\begin{array}{l}\text { Unable to detect active } \\
\text { colitis }\end{array}$ & $\begin{array}{l}98 \%-100 \% \\
{[27,28]}\end{array}$ & $\begin{array}{l}96 \%-99 \% \\
{[27,28]}\end{array}$ \\
\hline Antigen testing & $\begin{array}{l}\text { Reasonable sensitivity and } \\
\text { specificity for active disease }\end{array}$ & $\begin{array}{l}\text { Semiquantitative, results } \\
\text { subjective }\end{array}$ & $\begin{array}{l}60 \%-100 \% \\
{[29-31]}\end{array}$ & $\begin{array}{l}83 \%-100 \% \\
{[29-31]}\end{array}$ \\
\hline Culture & High specificity & $\begin{array}{l}\text { Poor sensitivity; long } \\
\text { incubation; }\end{array}$ & $\begin{array}{l}45 \%-78 \% \\
{[30,32]}\end{array}$ & $\begin{array}{l}89 \%-100 \% \\
{[27,30]}\end{array}$ \\
\hline DNA PCR (Serum) & $\begin{array}{l}\text { High negative predictive } \\
\text { value, may correlate with } \\
\text { active disease }\end{array}$ & $\begin{array}{l}\text { Different assays and } \\
\text { quantitation methods; low } \\
\text { specificity }\end{array}$ & $\begin{array}{l}65 \%-100 \% \\
{[29,30,33]}\end{array}$ & $\begin{array}{l}40 \%-92 \% \\
{[30,33,34]}\end{array}$ \\
\hline Histologic H\&E stain & Inexpensive stain & Poor sensitivity & $\begin{array}{l}10 \%-87 \% \\
{[35]}\end{array}$ & $\begin{array}{l}92 \%-100 \% \\
{[35]}\end{array}$ \\
\hline $\begin{array}{l}\text { Histologic IHC } \\
\text { staining }\end{array}$ & $\begin{array}{l}\text { Improves sensitivity over } \\
\text { H\&E staining }\end{array}$ & Expensive stain & $78 \%-93 \%[35]$ & $92 \%-100 \%[35]$ \\
\hline
\end{tabular}

demonstrated to improve clinical outcomes. A case study and review of the existing literature showed that among 26 total UC patients with CMV infection, the 20 untreated patients carried a $67 \%$ colectomy rate and $33 \%$ mortality [2]. In contrast, 6 out of 6 patients who were treated with antiviral therapy survived and avoided colectomy [2]. Similarly, a case control study found that among 10 UC patients with CMV infection, 2 patients treated with IV gangciclovir demonstrated resolution of symptoms and avoidance of surgery, whereas 6 of 8 untreated patients proceed to surgery [10]. Another study also reported reduced morbidity and mortality with antiviral therapy among 7 IBD patients with CMV infection (5 of whom were UC patients) [22]. The 5 of 5 treated patients went into remission with IV antiviral therapy, whereas the 2 patients with CMV who did not undergo antiviral therapy failed to improve and underwent colectomy [22].

Overall, these studies all suggest that CMV infection in the setting of ulcerative colitis imparts an increased morbidity and mortality rate, which can be decreased with the use of antiviral therapy.

\section{CMV Infection as an Innocent Bystander}

There are studies suggesting that the presence of CMV represents tropism towards areas of dysplasia and inflammation rather than a reflection of true disease. Proponents of the "innocent bystander" hypothesis believe that the presence of CMV does not contribute to increased morbidity and mortality beyond that of the underlying disease, and that antiviral therapy may eradicate the virus in this secondary, minor infectious state, without altering the course of underlying active UC disease [10]. One study demonstrated no difference in remission and colectomy rates between UC patients with CMV reactivation and nonCMV UC patients [39]. The investigators concluded that CMV reactivation has little effect on clinical course of UC patients [39]. However, this study was limited to patients with serologic evidence of CMV reactivation without demonstrated evidence of CMV on histologic examination. Another report concluded that the presence of CMV or antiviral therapy did not alter the clinical course of UC patients with CMV reactivation [40]. However, in this study 3 of 3 patients with CMV infection proceeded to urgent colectomy, and the one patient who was treated for CMV received IV acyclovir rather than ganciclovir or forscarnet [40]. Another reason for CMV infection as an "innocent bystander" was that CMV-positive patients who did not receive antiviral therapy following proctocolectomy did not show endoscopic or histological cytomegalovirus reactivation in the ileoanal pouch and in the remaining bowel [21]. However, surgery itself removed diseased bowel containing active CMV infection, and these patient were not on continued corticosteroid therapy postoperatively.

Even though there are reports indicating that CMV reactivation in UC patients may not affect the outcome of UC patients, it is the authors' opinion that UC patients found to have CMV reactivation should be treated with IV antiviral therapy, including ganciclovir or forscarnet, to reduce morbidity and mortality.

\section{Stratifying UC Patients with CMV Reactivation}

The true interplay between ulcerative colitis and CMV infection likely resides between these two competing theories of pathogenicity versus "innocent bystander." CMV virus indeed has a propensity towards colonizing the inflamed, dysplastic, granulated ulcer beds that are a hallmark of ulcerative colitis. In cases of lower viral burden, CMV infection may not meaningfully contribute to active colitis symptoms. However, the virus' proclivity toward infecting endothelial cells and inflamed ulcer beds, suggests that higher disease burden of CMV may actually lead to microvascular ischemia, elevated inflammatory response, and altered hemostasis, possibly exacerbating the existing vulnerable inflamed areas and leading to increased morbidity and mortality, beyond that of the underlying UC alone [19]. Clinical or laboratory markers that can stratify whether CMV is a significant contributor to a patient's clinical scenario have yet to be identified. Furthermore, the challenge of determining which patients may benefit from antiviral 
therapy, immunosuppression escalation, or possible early surgical intervention still remains.

In prior studies, the density of cells infected with CMV has been shown to be associated with clinically relevant infection and severity of disease [19]. Recently, our group has presented similar findings that the severity of CMV involvement on histologic examination correlates well with both clinical outcomes and antiviral therapy response [41]. In this study of 43 IBD patients, 26 of which had UC, patients stratified to "highgrade infection," defined by presence of viral inclusion bodies on both routine $\mathrm{H} \& \mathrm{E}$ and special IHC stains, demonstrated a significantly higher colectomy rate $(57 \%)$ compared to patients stratified as "low-grade infection" (30\%), defined by the absence of viral inclusion bodies on routine $\mathrm{H} \& \mathrm{E}$ stains but the presence of positive CMV IHC stains against CMV monoclonal antigens [41]. In addition, the low-grade CMV group demonstrated a nearly identical colectomy rate between those treated and untreated with antiviral therapy (31\% versus $29 \%$ ). In contrast, the high-grade CMV group demonstrated a trend towards a decreased colectomy rate in those treated versus untreated with antiviral therapy (44\% versus $83 \%$ ) [41]. We propose that in patients with high-grade CMV infection on histology, CMV may be the main drive for gut inflammation, and antiviral therapy is effective in treating the inflammatory process and avoiding colectomy in these patients. In contrast, among IBD patients with low-grade CMV infection, the underlying IBD may be the main drive for gut inflammation, in which case treating IBD using immunosuppressants may improve clinical outcome.

In addition to determining which patients may respond to antiviral therapy, numerous unanswered questions remain regarding the relationship between CMV infection and ulcerative colitis. Thus far, an overwhelming majority of studies involve patients either hospitalized or evaluated for symptomatic disease, and the rate of CMV reactivation in quiescent UC disease is largely unstudied. Studies have suggested an increased rate of CMV reactivation in UC compared to Crohn's disease patients, but formal studies that examine the underlying etiology for this, as well as the difference in rate, outcome, and response to therapy are lacking.

\section{Final Conclusions}

Despite arguments of the nonpathogenicity of CMV reactivation, the overall existing literature indicates that UC patients are at increased risk for CMV reactivation, and that the failure to appropriately diagnose and treat these patients in a timely manner leads to significant morbidity and mortality. This increased risk is likely multifactorial due in part to both iatrogenic immunosuppression and underlying factors related to severe inflammation, possible malnutrition, and inherent immunogenicity. However, these underlying factors have not been fully elucidated and are not well understood.

Patients presenting with severe and/or refractory disease, especially patients with significant steroid use history should be evaluated for possible CMV reactivation as a possible contributor to their symptoms. Laboratory values can indicate prior exposure and may suggest active disease, but ultimately diagnosis depends on histologic examination of involved tissue demonstrating typical CMV viral inclusion bodies. In addition, special IHC staining should be performed on tissue samples to increase the sensitivity of diagnosis.

Patients with severe symptoms and evidence of active CMV on histologic examination should be treated promptly with appropriate intravenous antiviral agents to avoid increased risks of colectomy and possible mortality. Intravenous ganciclovir has been the agent that most experts endorse, but dosages and duration of therapy have not been established. Those not responding to IV antivirals are recommended to proceed to surgical intervention.

There remain many unresolved questions regarding the management of CMV reactivation in ulcerative colitis that need to be addressed with large, controlled studies.

\section{Abbreviations}

UC: Ulcerative Colitis

CMV: Cytomegalovirus

IHC: Immunohistochemistry

TNF: Tumor Necrosis Factor

H\&E: Haematoxylin and Eosin

IFN: Interferon.

\section{Conflict of Interests}

Then authors declare no financial or commercial conflict of interest.

\section{Acknowledgments}

The authors thank Stephan R. Targan, MD, for support and intellectual discussions and Cindy Ting, PharmD, for critical reading of the paper. This work was supported by grants from Inflammatory Bowel Disease and Immunobiology Research Institute (DQS).

\section{References}

[1] G. H. Taylor, "Cytomegalovirus," American Family Physician, vol. 67, no. 3, pp. 519-526, 2003.

[2] D. G. Begos, R. Rappaport, and D. Jain, "Cytomegalovirus infection masquerading as an ulcerative colitis flare- up: case report and review of the literature," Yale Journal of Biology and Medicine, vol. 69, no. 4, pp. 323-328, 1997.

[3] E. Dimitroulia, N. Spanakis, A. E. Konstantinidou, N. J. Legakis, and A. Tsakris, "Frequent detection of cytomegalovirus in the intestine of patients with inflammatory bowel disease," Inflammatory Bowel Diseases, vol. 12, no. 9, pp. 879-884, 2006.

[4] P. J. Hamlin, M. N. Shah, N. Scott, J. I. Wyatt, and P. D. Howdle, "Systemic cytomegalovirus infection complicating ulcerative colitis: a case report and review of the literature," Postgraduate Medical Journal, vol. 80, no. 942, pp. 233-235, 2004. 
[5] W. J. Britt and C. A. Alford, "Cytomegalovirus," Virology, vol. 2, no. 3, pp. 2493-2523, 1996.

[6] C. Soderberg-Naucler, "Human cytomegalovirus persists in its host and attacks and avoids elimination by the immune system," Critical Reviews in Immunology, vol. 26, no. 3, pp. 231-263, 2006.

[7] C. Soderberg-Naucler, K. N. Fish, and J. A. Nelson, "Reactivation of latent human cytomegalovirus by allogeneic stimulation of blood cells from healthy donors," Cell, vol. 91, no. 1, pp. 119-126, 1997.

[8] C. Soderberg-Naucler, K. N. Fish, and J. A. Nelson, "Interferon- $\gamma$ and tumor necrosis factor- $\alpha$ specifically induce formation of cytomegalovirus-permissive monocyte-derived macrophages that are refractory to the antiviral activity of these cytokines," Journal of Clinical Investigation, vol. 100, no. 12, pp. 3154-3163, 1997.

[9] E. Domenech, R. Vega, I. Ojanguren et al., "Cytomegalovirus infection in ulcerative colitis: a prospective, comparative study on prevalence and diagnostic strategy," Inflammatory Bowel Diseases, vol. 14, no. 10, pp. 1373-1379, 2008.

[10] N. Kambham, R. Vij, C. A. Cartwright, and T. Longacre, "Cytomegalovirus infection in steroidrefractory ulcerative colitis: a case-control study," American Journal of Surgical Pathology, vol. 28, no. 3, pp. 365-373, 2004.

[11] R. Osaki, A. Andoh, T. Tsujikawa et al., "Acute cytomegalovirus infection superimposed on corticosteroidnaive ulcerative colitis," Internal Medicine, vol. 47, no. 14, pp. 1341-1344, 2008.

[12] P. Pfau, M. L. Kochman, E. E. Furth, and G. R. Lichtenstein, "Cytomegalovirus colitis complicating ulcerative colitis in the steroid-naive patient," American Journal of Gastroenterology, vol. 96, no. 3, pp. 895-899, 2001.

[13] C. Rachima, E. Maoz, S. Apter et al., "Cytomegalovirus infection associated with ulcerative colitis in immunocompetent individuals," Postgraduate Medical Journal, vol. 74, no. 874, pp. 486-489, 1998.

[14] K. L. Streetz, T. Buhr, H. Wedemeyer et al., "Acute CMV-colitis in a patient with a history of ulcerative colitis," Scandinavian Journal of Gastroenterology, vol. 38, no. 1, pp. 119-122, 2003.

[15] Y. Takahashi and T. Tange, "Prevalence of cytomegalovirus infection in inflammatory bowel disease patients," Diseases of the Colon and Rectum, vol. 47, no. 5, pp. 722-726, 2004.

[16] K. A. Papadakis, J. K. Tung, S. W. Binder et al., "Outcome of cytomegalovirus infections in patients with inflammatory bowel disease," American Journal of Gastroenterology, vol. 96, no. 7, pp. 2137-2142, 2001.

[17] R. Vega, X. Bertran, M. Menacho et al., "Cytomegalovirus infection in patients with inflammatory bowel disease," American Journal of Gastroenterology, vol. 94, no. 4, pp. 1053-1056, 1999.

[18] V. C. Mariguela, S. G. F. Chacha, A. D. A. Cunha, L. E. D. A. Troncon, S. Zucoloto, and L. T. M. Figueiredo, "Cytomegalovirus in colorectal cancer and idiopathic ulcerative colitis," Revista do Instituto de Medicina Tropical de Sao Paulo, vol. 50, no. 2, pp. 83-87, 2008.

[19] A. Kuwabara, H. Okamoto, T. Suda, Y. Ajioka, and K. Hatakeyama, "Clinicopathologic characteristics of clinically relevant cytomegalovirus infection in inflammatory bowel disease," Journal of Gastroenterology, vol. 42, no. 10, pp. 823829, 2007.

[20] M. Minami, M. Ohta, T. Ohkura et al., "Cytomegalovirus infection in severe ulcerative colitis patients undergoing continuous intravenous cyclosporine treatment in Japan,"
World Journal of Gastroenterology, vol. 13, no. 5, pp. 754-760, 2007.

[21] G. Maconi, E. Colombo, P. Zerbi et al., "Prevalence, detection rate and outcome of cytomegalovirus infection in ulcerative colitis patients requiring colonic resection," Digestive and Liver Disease, vol. 37, no. 6, pp. 418-423, 2005.

[22] M. Cottone, G. Pietrosi, G. Martorana et al., "Prevalence of cytomegalovirus infection in severe refractory ulcerative and Crohn's colitis," American Journal of Gastroenterology, vol. 96, no. 3, pp. 773-775, 2001.

[23] V. D’Ovidio, P. Vernia, G. Gentile et al., “Cytomegalovirus infection in inflammatory bowel disease patients undergoing anti-TNF $\alpha$ therapy," Journal of Clinical Virology, vol. 43, no. 2, pp. 180-183, 2008.

[24] L. C. Hookey, W. Depew, A. Boag, and S. J. Vanner, "6Mercaptopurine and inflammatory bowel disease: hidden ground for the cytomegalovirus," Canadian Journal of Gastroenterology, vol. 17, no. 5, pp. 319-322, 2003.

[25] J. Kishore, U. Ghoshal, U. C. Ghoshal et al., "Infection with cytomegalovirus in patients with inflammatory bowel disease: prevalence, clinical significance and outcome," Journal of Medical Microbiology, vol. 53, no. 11, pp. 1155-1160, 2004.

[26] T. Kojima, T. Watanabe, K. Hata, M. Shinozaki, T. Yokoyama, and H. Nagawa, "Cytomegalovirus infection in ulcerative colitis," Scandinavian Journal of Gastroenterology, vol. 41, no. 6, pp. 706-711, 2006.

[27] M. G. Revello and G. Gerna, "Diagnosis and management of human cytomegalovirus infection in the mother, fetus, and newborn infant," Clinical Microbiology Reviews, vol. 15, no. 4, pp. 680-715, 2002.

[28] B. Weber, A. Berger, and H. Rabenau, "Human cytomegalovirus infection: diagnostic potential of recombinant antigens for cytomegalovirus antibody detection," Journal of Virological Methods, vol. 96, no. 2, pp. 157-170, 2001.

[29] A. T. Rowshani, F. J. Bemelman, E. M. M. van Leeuwen, R. A. W. Van Lier, and I. J. M. Ten Berge, "Clinical and immunologic aspects of cytomegalovirus infection in solid organ transplant recipients," Transplantation, vol. 79, no. 4, pp. 381-386, 2005.

[30] G. Boivin, J. Handfield, E. Toma et al., "Evaluation of the AMPLICOR cytomegalovirus test with specimens from human immunodeficiency virus-infected subjects," Journal of Clinical Microbiology, vol. 36, no. 9, pp. 2509-2513, 1998.

[31] T. Lazzarotto, G. T. Maine, P. Dal Monte, H. Frush, K. Shi, and M. P. Landini, "Detection of serum immunoglobulin $\mathrm{M}$ to human cytomegalovirus by Western blotting correlates better with virological data than detection by conventional enzyme immunoassay," Clinical and Diagnostic Laboratory Immunology, vol. 3, no. 5, pp. 597-600, 1996.

[32] J. D. Meyers, P. Ljungman, and L. D. Fisher, "Cytomegalovirus excretion as a predictor of cytomegalovirus disease after marrow transplantation: importance of cytomegalovirus viremia," Journal of Infectious Diseases, vol. 162, no. 2, pp. 373-380, 1990.

[33] A. Michaelides, L. Liolios, E. M. Glare et al., "Increased human cytomegalovirus (HCMV) DNA load in peripheral blood leukocytes after lung transplantation correlates with HCMV pneumonitis," Transplantation, vol. 72, no. 1, pp. 141-147, 2001.

[34] W. D. Rawlinson, "Broadsheet. Number 50: diagnosis of human cytomegalovirus infection and disease," Pathology, vol. 31, no. 2, pp. 109-115, 1999.

[35] L. Beaugerie, C. Cywiner-Golenzer, L. Monfort et al., "Definition and diagnosis of cytomegalovirus colitis in patients 
infected by human immunodeficiency virus," Journal of Acquired Immune Deficiency Syndromes and Human Retrovirology, vol. 14, no. 5, pp. 423-429, 1997.

[36] A. Kandiel and B. Lashner, "Cytomegalovirus colitis complicating inflammatory bowel disease," American Journal of Gastroenterology, vol. 101, no. 12, pp. 2857-2865, 2006.

[37] M. M. Maher and M. I. Nassar, "Acute cytomegalovirus infection is a risk factor in refractory and complicated inflammatory bowel disease," Digestive Diseases and Sciences, vol. 54, no. 11, pp. 2456-2462, 2009.

[38] V. Criscuoli, M. R. Rizzuto, and M. Cottone, "Cytomegalovirus and inflammatory bowel disease: is there a link?" World Journal of Gastroenterology, vol. 12, no. 30, pp. 4813-4818, 2006.

[39] K. Matsuoka, Y. Iwao, T. Mori et al., "Cytomegalovirus is frequently reactivated and disappears without antiviral agents in ulcerative colitis patients," American Journal of Gastroenterology, vol. 102, no. 2, pp. 331-337, 2007.

[40] I. A. Eyre-Brook and S. Dundas, "Incidence and clinical significance of colonic cytomegalovirus infection in idiopathic inflammatory bowel disease requiring colectomy," Gut, vol. 27, no. 12, pp. 1419-1425, 1986.

[41] M. Nguyen, K. Bradford, and X. Zhang, "Severity of cytomegalovirus infection on pathology specimens is associated with colectomy rates and response to antiviral therapy in inflammatory bowel disease patients," Digestive Disease Week, vol. 2010, no. 445, 2010. 


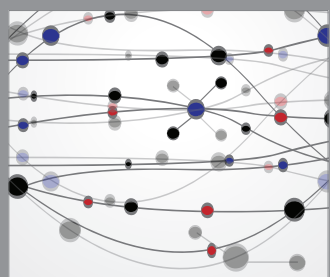

The Scientific World Journal
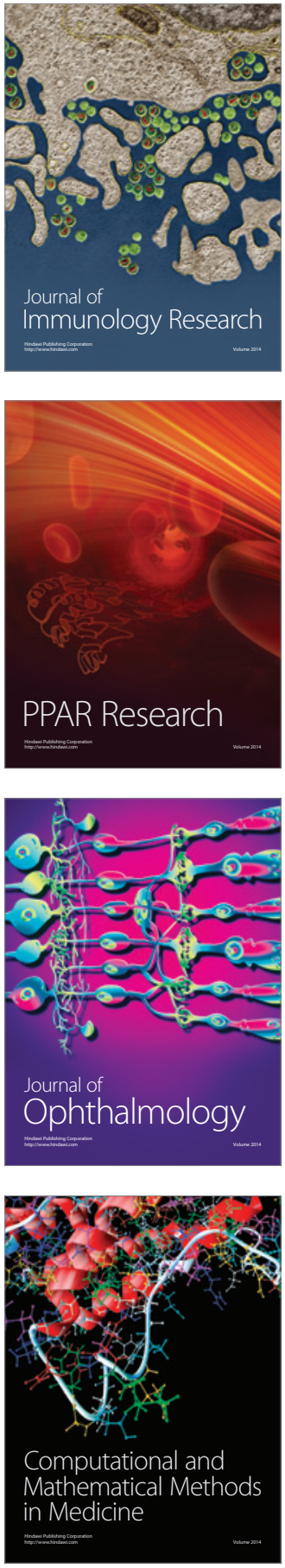

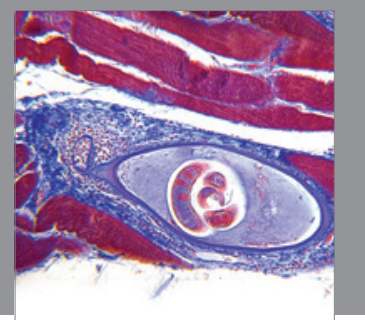

Gastroenterology

Research and Practice
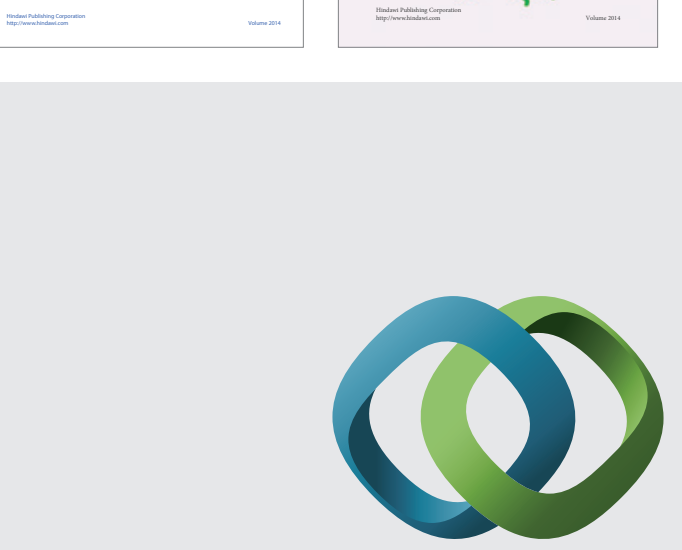

\section{Hindawi}

Submit your manuscripts at

http://www.hindawi.com
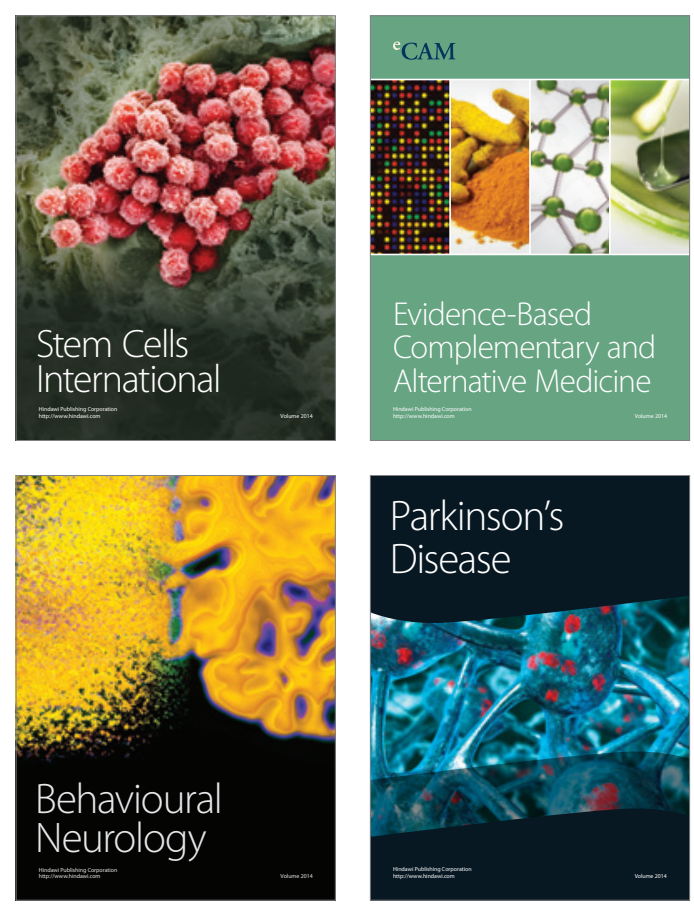

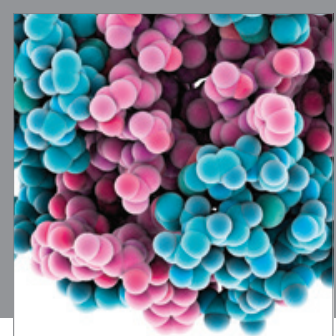

Journal of
Diabetes Research

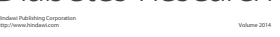

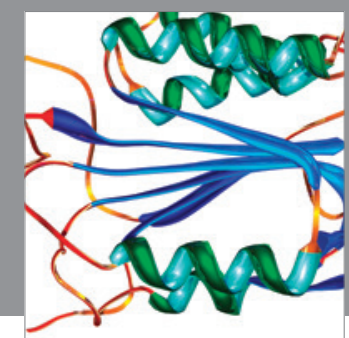

Disease Markers
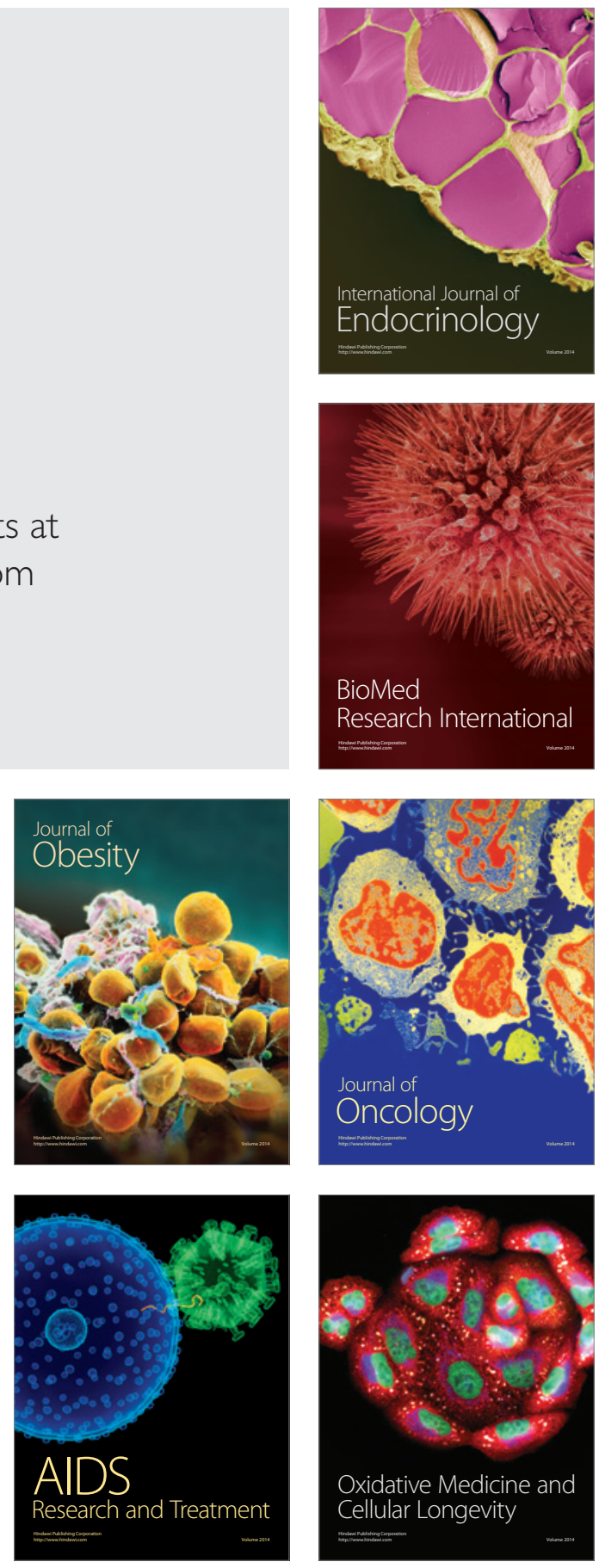